\title{
Dynamic Mode Decomposition on LES Result of Cylinder Cascade Wake
}

\author{
Tong Wang \\ School of Mechanical Engineering, Shanghai Jiao Tong University \\ 800 Dongchuan Road, Shanghai, China \\ twang@sjtu.edu.cn
}

\begin{abstract}
Flow around cylinder cascade is a common flow configuration which can be used in heat transfer and other industries. The interaction of vortices has an unavoidable impact on the downstream. The information of transient flow can be acquired by large eddy simulation(LES). In this paper, the cylinder cascade with the gap ratio of 2.0 was simulated by large eddy simulation method at two Reynolds number conditions and the mode decomposition algorithm was adopted to analyze the movement of vortex. Compared with information extracted by POD algorithm and FFT method, the validation of DMD algorithm was discussed by the results from the hypothetic scalar field and the experimental data. The accuracy of LES results and the characteristic of the vortex were analyzed by DMD algorithm. It has been verified that DMD algorithm can be used for cascade engineering on account of the accurate description of the flow structure.
\end{abstract}

Keywords: Large eddy simulation, cylinder cascade, dynamic mode decomposition, vortex structure

\section{Introduction}

Cascade flow is one of the common flow configurations in industry such as the blade cascade in axial compressor[1], the radial vaned diffuser of a centrifugal compressor[2] and the series of tubes in heat exchanger[3]. The cascade often exists as a component of the system, playing a significant role by wake interaction in the performance of the system, where the cylinder cascade, the simplest flow configuration, has been extensively discussed.

The discussions before usually regarded the cylinder cascade as an array of cylinders and characteristics related to the cylinder were posed. Awale[4] discussed the complex flow phenomenon behind four cylinders in side by side arrangement (cylinder cascade) at different gap-to-diameter (S/D) by studying the lift force signals. The secondary frequency which represents the interaction among the cylinders was found. The flow patterns caused by the wake interaction among the cylinders were revealed by the experiment and the numerical simulation[5-7]. The biased flow, spontaneous flopping flow (changing the direction from one side to another), neither forced nor spontaneous flopping flow and other flow patterns indicate that the flow around the cylinder cascade is different from the flow around a single cylinder due to multiple passages in the cylinder cascade.

The accurate description of the evolution of the wake behind the cylinder cascade poses a great challenge and the mode decomposition technologies are becoming more and more common in the analysis on turbulent flow. The key of the mode decomposition technologies is to extract the coherent structure which may describe the underlying physical mechanism of the turbulent flow. Proper Orthogonal Decomposition(POD), a mathematical method based on the data dimension reduction, was used to identify the coherent structures in turbulent flow [8]. It is an effective way to extract the dominant structures in turbulent flow based on the turbulent energy rank. Feng[9] used POD method to acquire the vortex dynamics of a circular cylinder controlled by a synthetic jet. The coefficient by POD and corresponding dominant frequency can reveal the characteristics of the flow mode. The second-order correlation function in POD algorithm causes the loss of information that may be necessary for the description of the transport process. Schmid[10]introduced a new mode decomposition method named Dynamic Mode Decomposition (DMD) to extract the dynamic information from a flow field at the basis of the Koopman analysis of nonlinear dynamical systems. Much work involving DMD algorithm has recently focused on the application to the different flow configurations such as the flow around high-speed-train[11] and cavity flow[12]. As for the flow around the cylinder, Tissot[13]adopted DMD to extract the flowing information of the 
vortex behind the cylinder at $\mathrm{Re}=13000$, Sakai[14]compared the results by DMD and POD at $\mathrm{Re}=500$. The flow structures based on Strouhal number(St) were investigated.

In this paper, air around the cylinder cascade at $\mathrm{Re}=1600$ and $\mathrm{Re}=10570$ was investigated numerically by LES method at $S / D=2$. Reynolds number is based on the free stream velocity $U_{\text {in }}$ and the diameter of the cylinders $D$. The validation of DMD algorithm were discussed by examining a hypothetic scalar field and the experimental data in the shear layer at $\mathrm{Re}=1600$. The mode decomposition on LES results was used to verify the accuracy of the numerical simulation method.

\section{Flow Field Domain and Numerical Method}

The computational domain of the cylinder cascade is shown in Fig. 1. There are five equidistant cylinders in side by side arrangement, where the cylinders on the periodic boundary are considered as a half in the domain. The diameter $D$ and height $H$ of the cylinder is the same value of $63.5 \mathrm{~mm}$ while $S$ (the center to center cylinder spacing) is equal to $2 D$. The upper and lower surfaces of the cylinders were fixed on the parallel walls. The upstream and downstream of the cylinders cascade extend respectively in $\mathrm{X}$ direction to acquire the fully developed turbulent flow.

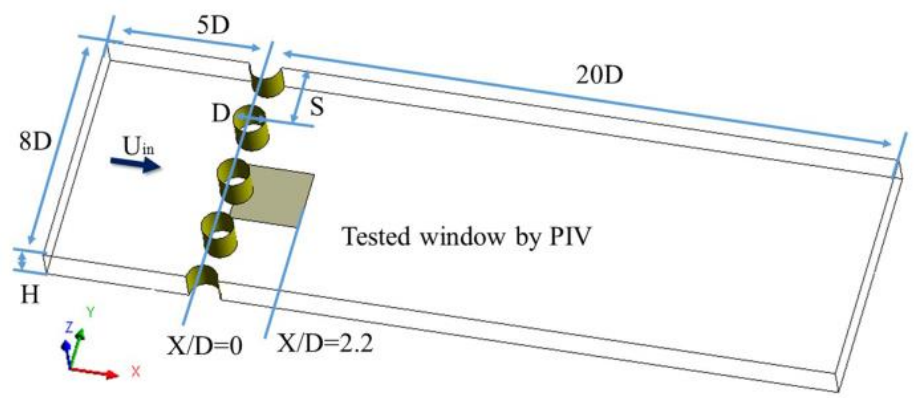

Fig. 1: Computational domain and tested window by PIV.

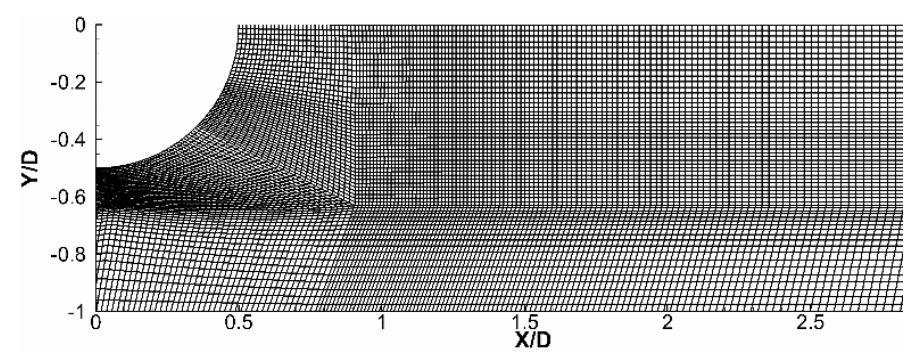

Fig. 2: Local grid behind the cylinder $(\mathrm{Re}=1600)$.

The flow field behind the center cylinder was tested by PIV technology[15]. The tested window is the middle section along the $\mathrm{Z}$ direction, shown as the marked rectangular in Fig. 1. The test region was $0<\mathrm{X} / \mathrm{D}<2.2$ and $1.0<\mathrm{Y} / \mathrm{D}<1.0$. At the inlet of the flow field, the constant velocity was set as the following: $\operatorname{Re}=1600, U_{i n}=0.373 \mathrm{~m} / \mathrm{s}$; $\operatorname{Re}=10570, U_{i n}=2.464 \mathrm{~m} / \mathrm{s}$. The outlet boundary condition was defined as the constant pressure of $1 \mathrm{~atm}$.

The wake flow behind the cascade is an unsteady flow with vortex shedding and interaction. The unsteady Reynolds average Navier-Stokes equations simulation (URANS) is a relatively cost-effective method to acquire the transient flow field behind the cascade. URANS method models turbulence through additional differential equations. In this paper, the non-negligible error still exists in shedding frequency of the URANS after the grid independence analysis. Compared to URANS, large eddy simulation (LES) resolves eddies which are larger than the grid size directly and models the sub-grid scale turbulence by turbulent model. LES method in ANSYS CFX software were used to accomplish the computation.

In LES, the filtering grid scale of the discrete equations is calculated by local grid. The proper grid distribution is necessary for a reasonable result. Zhang[16] proposed an adaptive control strategy based on turbulent energy ratio. The refined grid distribution is shown in Fig. 2, which is based on the energy ratio coefficient of 30\%, (i.e. turbulent dissipation energy and inertia sub-region is about $30 \%$ in the energy spectrum distribution of the flow field. )The grid distribution was symmetrical to the centerline along $\mathrm{X}$ direction and the grids between the adjacent cylinders were also 
refined to capture the wake interaction accurately. The total grid number was 5,000,000 for $\operatorname{Re}=1600$. As for $\operatorname{Re}=10570$, the total grid number, based on the energy ratio of $40 \%$, was $9,000,000$.

The dimensionless numerical time step $\left(\Delta t^{+}=\Delta t U_{\text {in }} / D\right)$ was set to 0.01 , where $\Delta t$ is the physical time step, to ensure an average CFL number of 0.5 . The initial flow field was from the converged result of URANS. The accuracy of the results by LES were verified by WANG[17].

\section{Model Decomposition Algorithms}

\subsection{Dynamic Mode Decomposition Algorithm}

The theory of dynamic mode decomposition (DMD) is from the koopman mode decomposition which describes the nonlinear flow by koopman operator. Arnoldi algorithm is used to compute koopman operator from snapshots. It can also produce approximations of koopman modes and related eigenvalues for dynamic mode decomposition.

In DMD algorithm, a sequence of data field $\mathbf{V}_{1}^{n}$, consisting of equi-spaced column vectors $\mathbf{v}_{i}$, can be written as

$$
\mathbf{V}_{1}^{n}=\left\{\mathbf{v}_{1}, \mathbf{v}_{2}, \cdots \mathbf{v}_{n}\right\}
$$

For experimental and numerical simulation results, the snapshots $\mathbf{v}_{i}$ are from a nonlinear system. A linear mapping $\mathbf{A}$ is adopted to describe the relationship between $\mathbf{v}_{i+1}$ and $\mathbf{v}_{i}$ by a linear tangent approximation. We can thus write:

$$
\mathbf{v}_{i+1}=\mathbf{A} \mathbf{v}_{i}
$$

The eigenvalues and eigenvectors of the system matrix $\mathbf{A}$ describe the underlying dynamic characteristics of the dynamic systems. The matrix A can be solved by Arnoldi method:

$$
\mathbf{V}_{2}^{n}=\mathbf{A} \mathbf{V}_{1}^{n-1} \approx \mathbf{V}_{1}^{n-1} \mathbf{S}
$$

The eigenvalues of $\mathbf{S}$ approximate some of the eigenvalues of the full matrix $\mathbf{A} \cdot \mathbf{V}_{1}^{n-1}$ can be decomposed into matrix $\mathbf{U}$, $\Sigma$ and $\mathbf{V}$ by using the singular value decomposition. The equation is expressed as:

$$
\mathbf{V}_{1}^{n-1}=\mathbf{U} \mathbf{\Sigma} \mathbf{V}^{T}
$$

Where $\mathbf{U}$ and $\mathbf{V}$ are the orthogonal matrix and $\Sigma$ is the diagonal matrix.

$$
\tilde{\mathbf{S}} \equiv \mathbf{U}^{T} \mathbf{A} \mathbf{U}=\mathbf{U}^{T} \mathbf{A}\left(\mathbf{U} \boldsymbol{\Sigma} \mathbf{V}^{T}\right) \mathbf{V} \boldsymbol{\Sigma}^{-1}=\mathbf{U}^{T} \mathbf{V}_{2}^{n} \mathbf{V} \boldsymbol{\Sigma}^{-1}
$$

Then the eigenvalues $\lambda_{i}$ and eigenvectors of $\mathbf{A}$ can be computed as:

$$
\tilde{\mathbf{S}} \mathbf{y}_{\mathbf{i}}=\lambda_{i} \mathbf{y}_{i}
$$

Finally, the dynamic modes are calculated as

$$
\mathbf{\Phi}_{i}=\mathbf{U y}_{i}
$$

The growth/decay rate $\sigma_{i}$ and the frequency $\omega_{\mathrm{i}}$ of the $i$-th mode of $\Phi_{i}$ can be calculated by using the logarithm of the eigenvalue $\lambda_{i}$ of each mode.

$$
\sigma_{i}=\operatorname{Re}\left[\ln \left(\lambda_{i}\right) / \Delta t\right] ; \quad \omega_{i}=\operatorname{Im}\left[\ln \left(\lambda_{i}\right)\right] / \Delta t / 2 \pi
$$


The energy of each mode can be decided by the norm of the modes $\left\|\Phi_{i}\right\|$. It provides an evaluation criterion to identify the dominant dynamical modes.

\subsection{Validation of DMD Algorithm}

The wake behind the cylinder cascade is caused by the alternate shedding eddies, and the high-order harmonics related to the Karman frequency can also be found in the wake. The signals in the wake appear in a sine waveform. According to the description above, the scalar field was provided as follow:

$$
\begin{gathered}
p=p_{0}+p_{1}+p_{2}+p_{3} \\
p_{0}(x, y, t)=\exp \left(-y^{2} / 0.7\right) \\
p_{n}(x, y, t)=\alpha_{n}(t) \exp \left[\left(a_{n} x+b_{n}\right) y^{2}\right] \cos \left(c_{n} \pi x+2 d_{n} \pi t\right) \quad n=1,2,3
\end{gathered}
$$

Where the scale of the structure is decided by $a_{n}$ and $b_{n}$, the variations of the structure in space and time are decided by $c_{n}$ and $d_{n}$, respectively. The growth or decay rate of the structure are decided by $\alpha_{n}(t)$. The hypothetic scalar field was

\begin{tabular}{|c|c|c|c|c|c|}
\hline & & $a_{n}$ & & & $d_{n}$ \\
\hline 1 & 2 & -10 & 2 & 3 & $\exp (t / 10)$ \\
\hline 2 & 5 & -30 & 3 & 7 & $\exp (-t / 50)$ \\
\hline 3 & 12 & -40 & 10 & 17 & $\exp (-t / 10)$ \\
\hline
\end{tabular}
composed of three different structures $(n=1,2$, and 3$)$. The parameters needed in expression (11) are shown in table 1.

Table 1: Parameters for the hypothetic scalar field.

The coprime frequencies of $3 \mathrm{~Hz}, 7 \mathrm{~Hz}$ and $17 \mathrm{~Hz}$ can be found in table 1 . The low-frequency structure has a growth rate of 0.1 while the high-frequency structures have the decay rate of 0.02 and 0.1 . The hypothetic scalar field was extracted by POD and DMD algorithm separately.

The spectrum distribution by DMD is shown in Fig. 3. The accurate growth/decay rate of the scalar field can be identified by DMD algorithm. The energy of the $2^{\text {nd }}$ mode and the $3^{\text {rd }}$ mode are similar in Fig. 3(b), and the modes extracted from the scalar field by DMD algorithm exactly reflect the hypothetic scalar field.

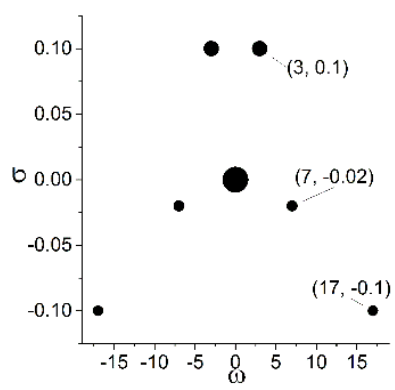

(a)Decomposition spectrum

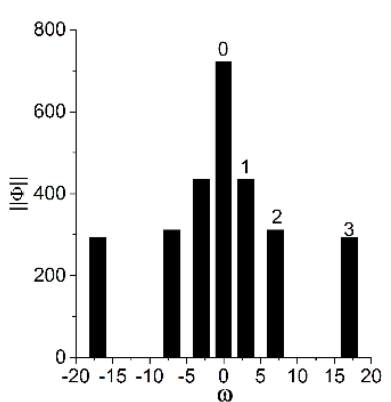

(b) Energy spectrum

Fig. 3: Spectrum by DMD algorithm. 


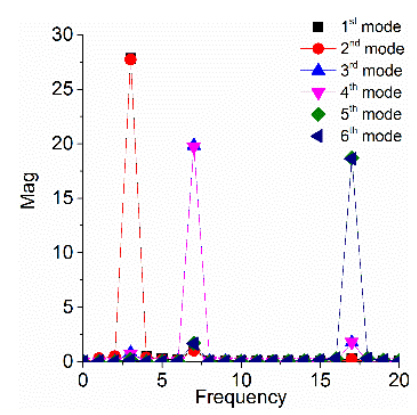

(a) Frequency of modes

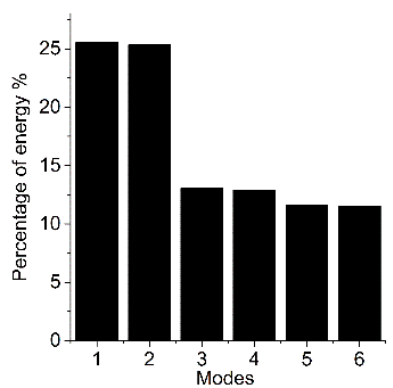

(b) Energy of each mode

Fig. 4: Modes by POD algorithm.

The model decomposition by POD algorithm is shown Fig. 4. 3-pair of modes are extracted. The frequency information of the modes is shown in Fig.4(a), where each mode is composed of at least two frequencies and $3 \mathrm{~Hz}$ occupies the dominant position in the $1^{\text {st }}$ mode and the $2^{\text {nd }}$ mode. Each pair has the dominant frequency value as that in table 1 . The energy of each mode by POD is shown in Fig.4(b). The first pair modes have higher energy while the other two pair modes have lower energy at the same degree.

The comparison between the results from DMD and POD is shown in Fig. 5. The modes extracted by DMD algorithm are shown in Fig.5(a). The modes accurately describe the movement of the structures in spatial and temporal. There is visible difference in the structure of the $4^{\text {th }}$ mode in POD compared with the hypothetic field in Fig.5(b). It is indicated that there are differences between the results from POD and DMD algorithm due to the different data processing method. The modes form POD algorithm are based on the spatial orthogonality while those from DMD algorithm are based on the temporal orthogonality. The modes are distinguished by energy in POD while by frequency in DMD. In other words, the mode by DMD contains a pure frequency while the mode by POD keeps multiple frequencies.

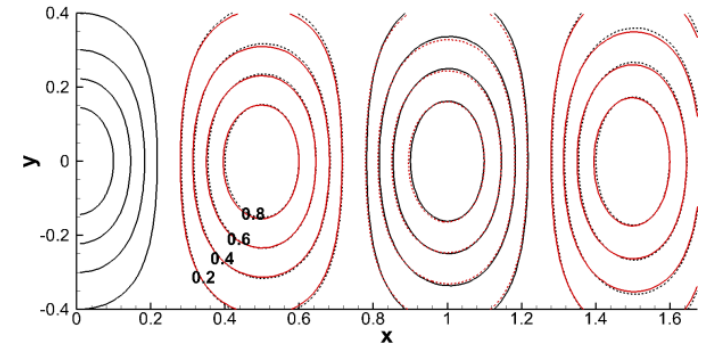

(a) $p_{1}$ componentand the modes extracted by DMD

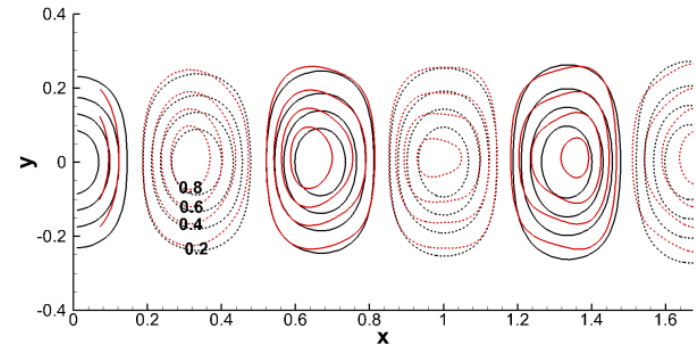

(b) Structure of the $4^{\text {th }}$ mode in POD

Fig. 5: Comparison between the component of the hypothetic field and model decomposition algorithm (black line: hypothetic field; red line: modes extracted by the algorithms).

Furthermore, the tested flow field in the shear layer of the cylinder cascade at $R e=1600$ was chosen to study the relationships between the frequency information from DMD and Fast Fourier Transform (FFT) method. FFT method has been widely used in the analysis of unsteady signal. The results obtained by DMD and FFT in the shear layer region are shown in Fig.6 and Fig. 7,the coordinate system is plotted as a log-log scale. Strouhal number, based on $U_{\text {in }}$ and $D$, represents the frequency information in the flow around the cylinder cascade. The norm of the modes by DMD is normalized in Fig.6.Different Strouhal numbers are found at the different points in the shear layer by FFT method, shown in Fig. 7. The dominant frequency of the shear eddies and Karman vortex discerned by DMD algorithm are shown in Fig.6. Otherwise, it begins to be clear at $1.5 \mathrm{X} / \mathrm{D}$ by FFT method.

The Kolmogorov power law (-5/3)[18] based on the isotropic turbulence hypothesisis also plotted (the oblique line) in Fig. 6 and Fig. 7. Generally, the region correspoding to the the Kolmogorov power law in energy spectrum is called inertial sub-range[19]. through DMD, the data agree well with the Kolmogorov power law which indicates that the modes extracted by DMD display a turbulent energy cascade. The inertial sub-range is not obvious at $0.5 \mathrm{X} / \mathrm{D}$ by FFT method.

Compared with the information by POD and FFT, the modes by DMD reflect the evolution of the flow structure in a large region in Fig.6 and DMD is more appropriate to be used to analyze the flow in the cylinder cascade wake. 


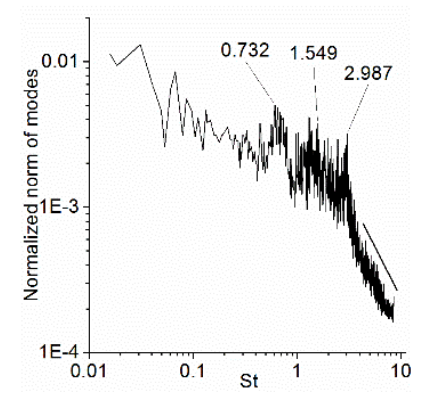

Fig. 6: Energy spectrum by DMD in shear layer region .
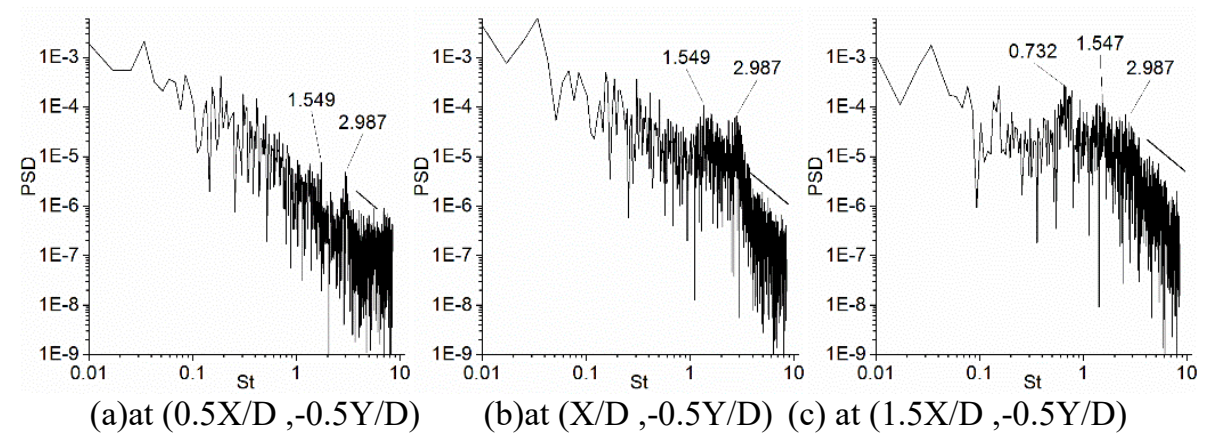

Fig. 7: Spectrum by FFT at a point.

\section{Application of DMD Algorithm}

\subsection{Experimental Data}

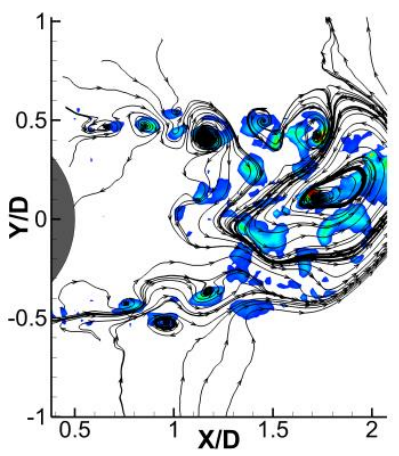

(a) $\mathrm{Re}=1600$

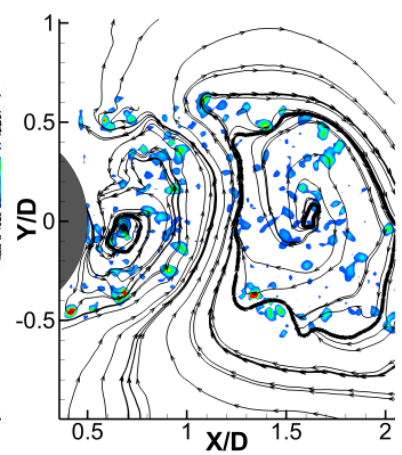

(b) $\mathrm{Re}=10570$

Fig. 8: Streamlines of experimental data.

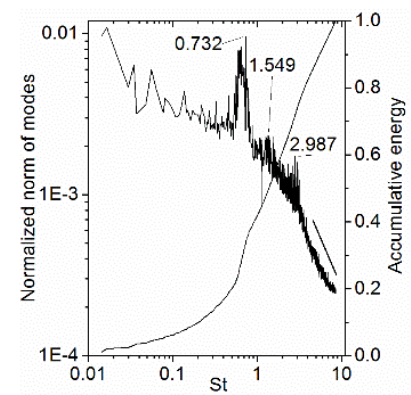

(a) $\mathrm{Re}=1600$

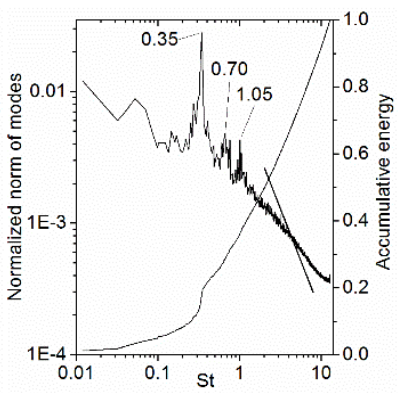

(b) $\mathrm{Re}=10570$

Fig. 9: Energy spectrum by DMD. 
The instantaneous streamlines, in which the mean flow is extracted are shown in Fig. 8 at different Reynolds numbers. The background of figure is coloured with Q criterion[20] which stands for the intensity of vortex. The coloured vortices formed a vortex chain along the shear layer region and it becomes a large turbulent eddy at the tail of the wake. The large turbulent eddy is closer to the cylinder and the diameter of shear vortex is smaller with increment of Reynolds number.

Two thousand pictures of instantaneous velocity field at the sampling time $\Delta t=0.01 \mathrm{~s}$ for $\mathrm{Re}=1600$ and $\Delta t=0.001 \mathrm{~s}$ for $\mathrm{Re}=10570$ were processed by DMD. The energy spectrums of DMD and Kolmogorov power law (-5/3) are shown in Fig.9. The inertial sub-region in high Reynolds number is wider and contains more energy. On the other hand, the flow behind the cylinder cascade at $\mathrm{Re}=10570$ is too drastic to be incompatible with isotropic turbulence hypothesis. The slope of the inetia sub-region changes from $-5 / 3$ to -0.7 in Fig. 9(b).

\subsection{LES Results}

The streamlines of mean flow by LES are shown in Fig. 10. The air passing the gap between two cylinders has a deflection phenomenon at $\mathrm{Re}=1600$, shown in Fig. 10(a). It results in a narrow and wide alternating wake behind the cylinder cascade, whereas it is not clear in the flow field at $\mathrm{Re}=10570$.

The sampling frequency was 12 times that of the shedding frequency. Rapid convergence was observed when 30 snapshots and 3 cycles of data were used. The domain for application of DMD was a full plane of the intermediate section in $\mathrm{Z}$ direction.

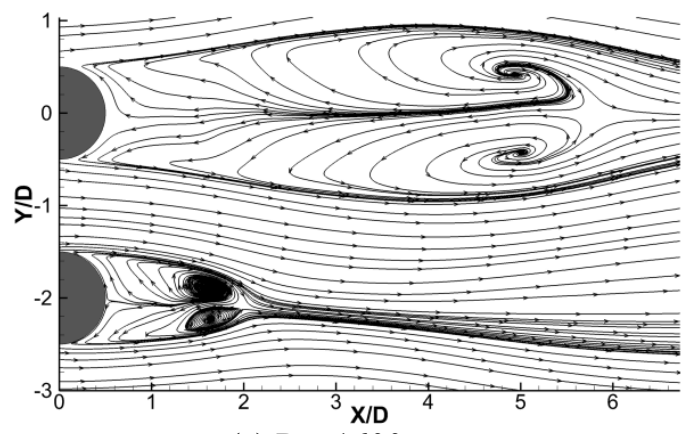

(a) $\mathrm{Re}=1600$

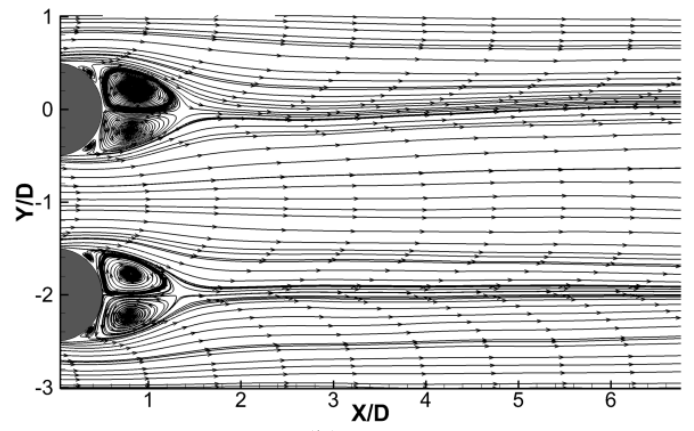

(b) $\mathrm{Re}=10570$

Fig. 10: Streamlines of average flow field by LES.

The structure of the Karman vortex extracted by DMD is shown in Fig. 11. At Re=1600, compared with those from experimental data, there is a small difference in the core of the Karman vortex on the shear layer. The Karman vortex detached from shear layers are coincident though the occurrence position of the Karman vortex by LES is far away from the cylinder. At $\mathrm{Re}=10570$, there is no obvious vortex found in the shear layer and the diameter of Karman vortex increased.

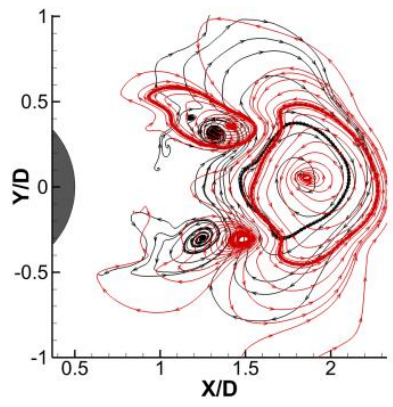

(a) $\operatorname{Re}=1600$

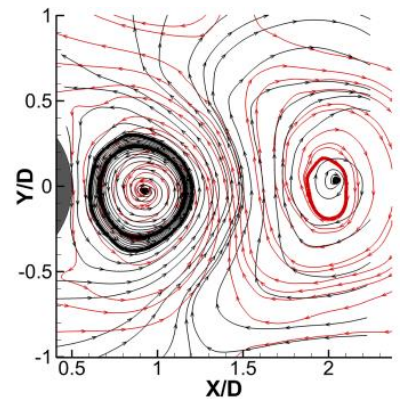

(b) $\operatorname{Re}=10570$

Fig. 11: Structure of Karman vortex (Black line: experimental data. Red line: LES results). 


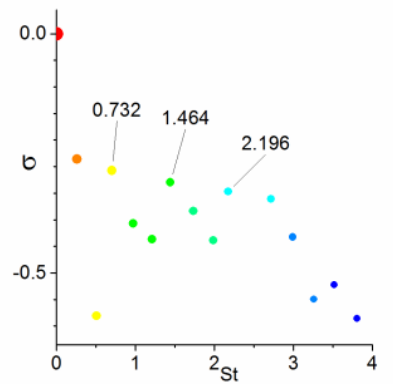

(a) $\operatorname{Re}=1600$

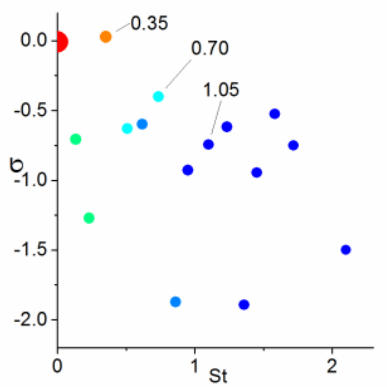

(b) $\operatorname{Re}=10570$

Fig. 12: Spectrum of DMD.

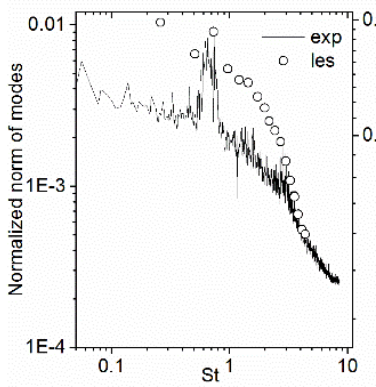

(a) $\mathrm{Re}=1600$

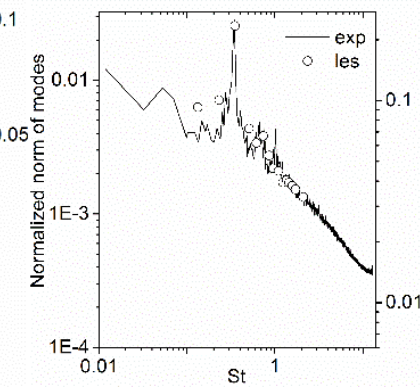

(b) $\mathrm{Re}=10570$

Fig. 13: Energy spectrum by DMD.

The spectrum from LES of each mode is shown in Fig. 12, where colours represent the mode energy. The modes with larger energy are coloured red. The mode $\mathrm{St}=0$ represents the mean flow and the decay rate is close to 0 . In Fig. 12(a), the shedding St Number 0.732 is the same as that from experimental data and the high-order harmonics such as 1.464 and 2.196 with low decay rate are found as well. The mode $\mathrm{St}=0.244$ also has large energy and low decay rate. As for $\mathrm{Re}=10570$, The modes $\mathrm{St}=0.35,0.7$ and 1.05 from LES are also equal to the experiment and the mode $\mathrm{St}=1.05 \mathrm{has}$ an obviously higher decay rate compared with the mode $\mathrm{St}=2.196$ at $\mathrm{Re}=1600$.

he energy spectrum of LES result is shown in Fig 13. The inertial sub-range of the energy spectrum is distinct and the Kolmogorov law of LES is similar to that of the experimental result at two Reynolds numbers. As mentioned in section 2, the generation of the grids is based on the energy ratio coefficient of $30 \%$ at $R e=1600$ and $40 \%$ at $R e=10570$. Despite the different analyzed region between LES results and experimental data, the limitation of the maximum resolution of St number in DMD. The energy ratio can still be estimated by LES result. About $80 \%(\mathrm{Re}=1600)$ and $55 \%(\mathrm{Re}=10570)$ of the total turbulent kinetic energy are captured by LES in Fig 9 and Fig 13. The grid generation method in this paper is reliable.

The decay/growth rate of the modes reflects the evolution of the corresponding coherent structure spatially and temporally. The decay rate of the modes in inertial sub-range is high. For the wake flow at $\operatorname{Re}=10570$, the decay rate of Karman vortex is closer to 0 compared with that at $\mathrm{Re}=1600$, which indicates that the evolution of Karman vortex behind the cylinder cascade will be more stable as Reynolds number increases. On the other hand, the other flow structures became more unstable. The detailed flow structures are discussed in Fig 14 and Fig 15.

Those cylinders in the computational domain are named for the convenience of analysis. The cylinder at $\mathrm{Y} / \mathrm{D}=0$ is named cylinder 1 , the cylinder at $\mathrm{Y} / \mathrm{D}=2$ is named cylinder 2 , the cylinder at $\mathrm{Y} / \mathrm{D}=-2$ is named cylinder 3 and the cylinder at the periodic boundary is named cylinder 4 .

The dimensionless value set as $\omega^{+}=\omega D / U_{\text {in }}$ is shown in Fig 14. The vorticity of the vortex shedding from cylinder 2 and cylinder 3 is attenuated and the vortex will deviate from the centerline of corresponding cylinder at $\mathrm{X} / \mathrm{D}=3$ in Fig 14(a). The modes in Fig 14(c) (d) are due to the interaction of the Karman vortex. The width of vortex in flow direction decreases with increasing frequency. When the flow field contains one cylinder, the structure of the vortex above has a reflection symmetry about the centerline $\mathrm{Y} / \mathrm{D}=0[21]$. In this paper, there are more than one cylinders in the flow field and the interaction of the harmonic mode between the adjacent cylinders is intensive. 
In Fig. 14(c) and Fig. 14(e), the regions marked with black circle represents the phenomenon that the contact of the vortex causes a change in the original trajectory. The interaction of the Karman vortex shedding from adjacent cylinders is shown in an intuitive way in Fig. 15(a). The red line shows that there are some eddies on the shear layer of cylinder 1 and its motion direction is parallel to the vortex shedding from cylinder 3. The field in red circle shows that the movement of eddies shedding from cylinder4 is blocked by eddies shedding from cylinder 3.

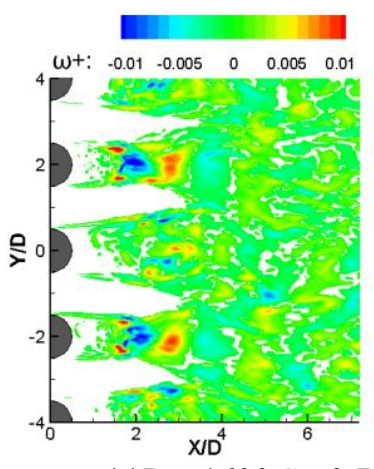

(a) $\mathrm{Re}=1600 \mathrm{St}=0.732$

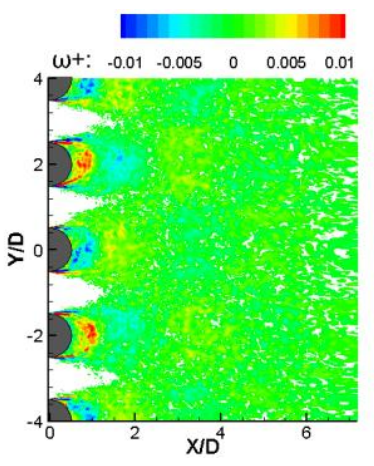

(b) $\mathrm{Re}=10570 \mathrm{St}=0.35$

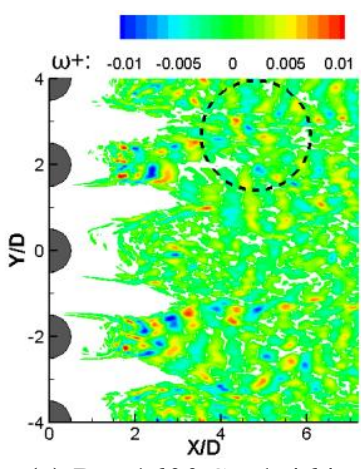

(c) $\mathrm{Re}=1600 \mathrm{St}=1.464$

Fig. 14: Vorticity contour.

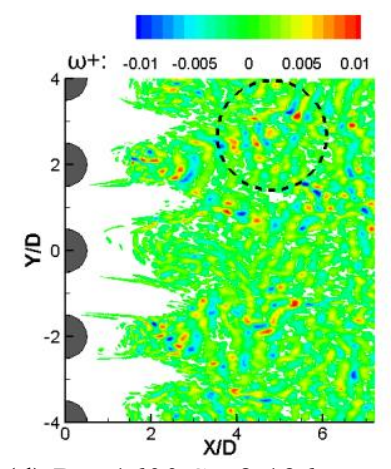

(d) $\mathrm{Re}=1600 \mathrm{St}=2.196$

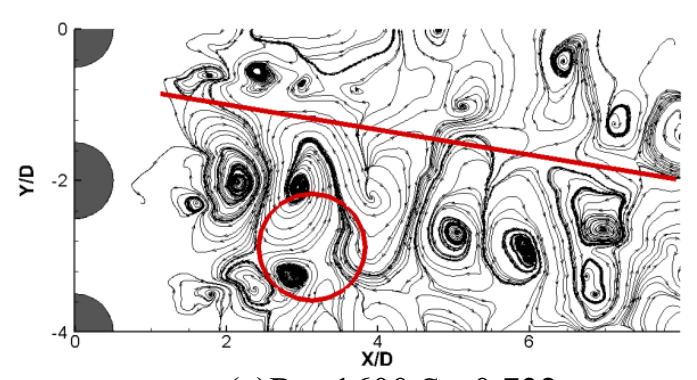

(a) $\mathrm{Re}=1600 \mathrm{St}=0.732$

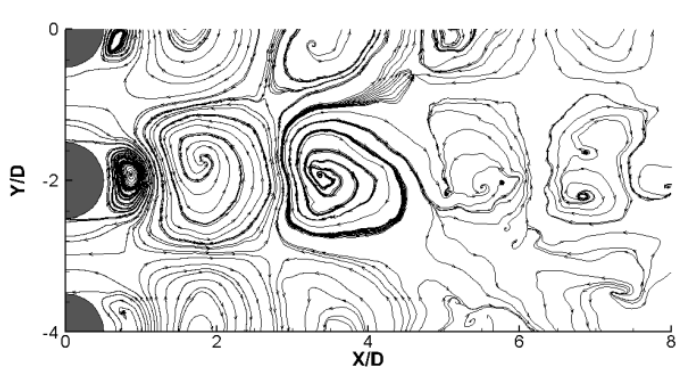

(b) $\mathrm{Re}=10570 \mathrm{St}=0.35$

Fig. 15: Streamlines of modes.

At $\mathrm{Re}=10570$, the difference between the adjacent cylinder on the structure of the Karman vortex disappear in Fig. 14.(b) and Fig. 15(b). The edge of the Karman vortex shedding from the adjacent cylinders begins to interact with each other at $\mathrm{X} / \mathrm{D}=1.5$ which makes the vortices difficult to deflect.

As mentioned earlier, the flow field is composed of vortices of different scales and different frequencies and the vortex can maintain intensity and structure for a long distance at $\mathrm{Re}=1600$. It seems that the vortices of higher frequency and smaller scale are embedded in the vortices of lower frequency and larger scale. As for $\mathrm{Re}=10570$, the intensity of vortex has a higher decay rate and the deformation of structures of vortex will happen at $X / D=4$. Finally, the flow field around cylinder cascade at $\mathrm{Re}=1600$ are more affected by the cylinders with wider wake. As for $\mathrm{Re}=10570$, the vortex shedding from cylinders is more easily dissipated and mixed with each other.

\section{Conclusion}

Two nonlinear dynamical flow fields $(\mathrm{Re}=1600, \mathrm{Re}=10570)$ were simulated by LES method. DMD algorithm has been presented to analyze the flow field behind cylinder cascade. It is verified that DMD algorithm is more appropriate to be used to get the dominant flow structure.

The modes extracted from DMD by frequency reflect the information of the flow field behind the cylinder cascade. The flow field behind the cascade is mainly occupied by the Karman vortex with high energy. As for Re=1600, Karman vortex and the corresponding high-order harmonics have smaller diameter and can move for longer distance. The encounter of the vortex results in the unstable flow behind the cascade. As for $\mathrm{Re}=10570$, the Karman vortex with larger diameter dissipates quickly. The straight-line motion of Karman vortex is stable and the decay rate is closer to 0 than $\mathrm{Re}=1600$. On the other hand, the other structures become unstable and have obvious high decay rate. 


\section{Acknowledgements}

The research is supported by National Natural Science Foundation of China under grant No.51276108. The author would like to thank for supporting the research work. The tested data were got from the Dr. Thole in Penn State University, the authors appreciate her help.

\section{References}

[1] R. J. Volino, "Effect of unsteady wakes on boundary layer separation on a very high lift low pressure turbine airfoil," Journal of Turbomachinery, vol. 134, pp. 011011, 2012.

[2] A. Marsan, et al, "Temporal behaviour of a corner separation in a radial vaned diffuser of a centrifugal compressor operating near surge," Journal of Thermal Science, vol. 22, pp. 555-564, 2013.

[3] J. K. Ostanek, K.A. Thole, "Wake development in staggered short cylinder arrays within a channel," Experiments in fluids, vol. 53, pp. 673-697, 2012.

[4] S. D. Awale, "Flow around row of four circular cylinders," J. Mater. Sci. Mech. Eng., vol. 1, pp. 11-17, 2014.

[5] H. Akilli, A. Akar, C. Karakus, "Flow characteristics of circular cylinders arranged side-by-side in shallow water," Flow measurement and Instrumentation, vol. 15, pp. 187-197, 2004.

[6] D. Guillaume, J. LaRue, "Investigation of the flopping regime with two-, three-and four-cylinder arrays," Experiments in Fluids, vol. 27, pp. 145-156, 1999.

[7] S. R. Kumar, A. Sharma, A. Agrawal, "Simulation of flow around a row of square cylinders," Journal of Fluid Mechanics, vol. 606, pp. 369-397, 2008.

[8] L. Sirovich, "Turbulence and the dynamics of coherent structures. I. Coherent structures," Quarterly of applied mathematics, vol. 45, pp. 561-571, 1987.

[9] L.-H. Feng, J.-J. Wang, C. Pan, "Proper orthogonal decomposition analysis of vortex dynamics of a circular cylinder under synthetic jet control," Physics of Fluids, vol. 23, pp. 014106, 2011.

[10] P. J. Schmid, "Dynamic mode decomposition of numerical and experimental data," Journal of fluid mechanics, vol. 656, pp. 5-28, 2010.

[11] T. W. Muld, G. Efraimsson, D.S. Henningson, "Flow structures around a high-speed train extracted using proper orthogonal decomposition and dynamic mode decomposition," Computers \& Fluids, vol. 57, pp. 87-97, 2012.

[12] A. Seena, H. J. Sung, "Dynamic mode decomposition of turbulent cavity flows for self-sustained oscillations," International Journal of Heat and Fluid Flow, vol. 32, pp. 1098-1110, 2011.

[13] G. Tissot, L. Cordier, N. Benard, B. R. Noack, "Model reduction using dynamic mode decomposition," Comptes Rendus Mécanique, vol. 342, pp. 410-416, 2014.

[14] M. Sakai, Y. Sunada, T. Imamura, K. Rinoie, "Experimental and Numerical Studies on Flow behind a Circular Cylinder Based on POD and DMD," Transactions of the Japan Society for Aeronautical and Space Sciences, vol. 58, pp. 100-107, 2015.

[15] J. K. Ostanek, K.A. Thole, "Flow field Measurements in a Single Row of Low Aspect Ratio Pin Fins," Journal of Turbomachinery, vol. 134, pp. 051034, 2012.

[16] B. Zhang, T. Wang, C. Gu, "An adaptive control strategy for proper mesh distribution in large eddy simulation," Journal of Hydrodynamics, Ser. B, vol. 22, pp. 865-870, 2010.

[17] T. Wang, S. Gao, "LES Analysis on Cylinder Cascade Flow Based on Energy Ratio Coefficient," HEFAT2016, Malaga, Spain, 2016.

[18] A. N. Kolmogorov, "The local structure of turbulence in incompressible viscous fluid for very large Reynolds numbers," in Dokl. Akad. Nauk SSSR, 1941.

[19] S. Pope, Turbulent Flows. Cambridge University Press, 2000.

[20] J. C. Hunt, A. A. Wray, P. Moin, "Eddies, streams, and convergence zones in turbulent flows," Center for Turbulence Research CTR-S 88, 1988.

[21] S. Bagheri, "Koopman-mode decomposition of the cylinder wake," Journal of Fluid Mechanics, vol. 726, pp. 596623, 2013. 